\title{
Sensing ligand binding to a clinically relevant lectin by tryptophan fluorescence anisotropy $\dagger$
}

\author{
Antonia Göhler, ${ }^{a}$ Claudia Büchner, ${ }^{a}$ Sabine André, ${ }^{b}$ Sören Doose, ${ }^{a}$ Herbert Kaltner ${ }^{* b}$ and H.-J. Gabius ${ }^{b}$ \\ Received 2nd August 2011, Accepted 26th September 2011 \\ DOI: $10.1039 / \mathrm{c} 1 \mathrm{an} 15692 \mathrm{f}$
}

\begin{abstract}
Increasing insights into the involvement of endogenous lectins in disease processes fuel the interest to develop potent inhibitors. As a consequence, robust assay procedures are required. Due to their activity as adhesion/growth-regulatory effectors this study focussed on galectins. The human proto-type galectin-1 was selected as representative of this family with conserved presence of a tryptophan moiety in the binding site. This structural feature was taken advantage of to establish its use as reporter for ligand contact measuring polarized fluorescence emission. The experimentally determined anisotropy $r_{0}$ was about 0.2 , altered by about $5 \%$ in the presence of the cognate disaccharide lactose. This parameter change enabled calculating the equilibrium binding constant and kinetic rate constants. The detailed analysis of the depolarization process further indicated fast conformational dynamics within the binding site. Since an inherent property of the protein was exploited, no labeling is needed. Owing to tryptophan's presence in carbohydrate-binding sites, also in other classes of lectins as well as in carbohydrate-binding modules and glycoenzymes (glycosyltransferases, glycosidases), this assay procedure can have relevance beyond galectins.
\end{abstract}

\section{Introduction}

The glycan chains have long been considered to primarily affect physicochemical properties of cellular glycoconjugates such as charge or solubility. However, insights into the sophistication of enzymatic glycan assembly and remodeling have paved the way for shaping the concept of the sugar code. ${ }^{1}$ One route toward the implied functional significance of glycan determinants is via interaction with specific receptors (lectins). ${ }^{2}$ In fact, they serve as translators of sugar-encoded messages, thereby triggering cellular responses such as adhesion, growth control or invasion/ migration. ${ }^{1,2}$ Documented association of lectin presence with disease manifestation and progression explains the growing interest in lectin-directed drug design. As a consequence, development of robust and sensitive ligand-binding assays is required. Mimicking the clinical context, both the lectin and the compound tested for binding capacity can be kept in solution. Under these conditions, a fluorescent label on the ligand can fulfil the given experimental prerequisite, and monitoring fluorescence polarization upon binding to a lectin is well established toward this

${ }^{a}$ Department of Biotechnology and Biophysics, Julius-Maximilians University, Am Hubland/Biozentrum, 97074 Wuerzburg, Germany

${ }^{b}$ Institute of Physiological Chemistry, Faculty of Veterinary Medicine, Ludwig-Maximilians University, Veterinaerstr. 13, 80539 Muenchen, Germany.E-mail: kaltner@lmu.de; Fax: +49 89 21802508; Tel: +49 89 21803984

$\dagger$ Electronic supplementary information: Table S1 and Fig. S1-S5. See DOI: $10.1039 / \mathrm{c} 1 \mathrm{an} 15692 \mathrm{f}$ end.,4 This approach underscores the versatility of using fluorescence parameters and turns attention to the respective potential of especially tryptophan (Trp) as intrinsic sensor. It is based on the comparatively large extinction coefficient $(\sim 5500$ $\mathrm{M}^{-1} \mathrm{~cm}^{-1}$ at $280 \mathrm{~nm}$ ) and a quantum yield between 10 and $20 \%$ depending on local environment and solvent conditions. ${ }^{5}$ Of further note, Trp offers a relatively large contact surface for $\mathrm{C}-\mathrm{H} / \pi$-interactions to galactose and other hexopyranoses, with its remarkable spatial flexibility for proper positioning of the sugar. ${ }^{6}$ Indeed, this feature is realized for sugar binding in lectins, carbohydrate-binding modules and glycoenzymes (glycosyltransferases, glycosidases) frequently, as reflected in the highest score of the sugar interface propensity for all 20 amino acids. ${ }^{2,7-9}$

The detection of increases in fluorescence polarization in the case of a plant lectin (wheat germ agglutinin) after binding $N$-acetylglucosamine ${ }^{10}$ as well as the structural and spectroscopic evidence for a Trp-galactose contact in galectins were the reasons to focus this model study on human galectin-1 (hGal1). ${ }^{11-14}$ This member of the family of adhesion/growth-regulatory galectins contains a single Trp residue in position 68 within the carbohydrate recognition domain (CRD). ${ }^{15,16} \mathrm{It}$ is of interest for a case study as target for inhibitor design, because it is involved in tumor invasion, e.g. in glioblastoma and pancreatic cancer, ${ }^{17,18}$ and in immune regulation on the level of $\mathrm{T}$ effector cells. ${ }^{19}$ Structural homology ensures the extrapolation of obtained data to the other family members. Thus, the spectral properties of hGal-1 were studied first and then the concept of using Trp as intrinsic sensor was put to the experimental test, under 
conditions (common equipment, small quantity of protein required) that will enable e.g. screening of compound libraries. $^{20,21}$ Besides validating the experimental approach, we report on fast conformational dynamics in the galectin's binding site by measuring Trp fluorescence anisotropy.

\section{Experimental}

Human Gal-1 was obtained by recombinant production, purified using affinity chromatography over lactosylated Sepharose 4B, prepared after divinylsulfone activation, as a crucial step and subjected to rigorous quality controls by one- and two-dimensional gel electrophoresis, mass spectrometric fingerprinting, circular dichroism spectroscopy, the latter to ascertain $\beta$-sandwich folding. ${ }^{22-24}$ Lectin activity was measured by haemagglutination using trypsin-treated and glutaraldehyde-fixed rabbit erythrocytes and cell binding/growth inhibition as well as solid-phase assays using the glycoprotein asialofetuin as ligand as described. ${ }^{21,25-28}$ Loss of activity owing to SH-group oxidation was minimized by exposing the lectin to a cysteine-modifying reagent (iodoacetamide) during elution from the affinity resin. ${ }^{29,30}$ Controls by isothermal titration calorimetry ensured the presence of one active binding site for lactose per subunit of the hGal-1 homodimer. ${ }^{15,31}$ All protein samples were stored at $-20^{\circ} \mathrm{C}$ after lyophilization and redissolved in phosphate-buffered saline (PBS) solution immediately before spectroscopical work was carried out.

Steady-state fluorescence emission and anisotropy measurements of the Trp signal used a commercial L-shaped fluorometer (FP-6500; Jasco GmbH, Gross-Umstadt, Germany). Absorption measurements were performed with a commercial UV/VIS double-beam spectrophotometer (V-650; Jasco GmbH). In all fluorescence measurements the protein concentration was kept below $20 \mu \mathrm{M}$ (corresponding to an optical density $<0.3$ at 280 $\mathrm{nm}$ ) in order to exclude reabsorption and Förster resonance energy transfer effects. In anisotropy measurements, the system set-up was manually selected by adjusting linear polarization filters transmitting either between 200 and $700 \mathrm{~nm}$ (UV polarizer) or between 320 and $750 \mathrm{~nm}$ (VIS polarizer). The excitation and emission bandwidths were $10 \mathrm{~nm}$. For each single measurement, the $G$ factor correcting for differences in detection of vertically and horizontally polarized light was determined by excitation with horizontally polarized light. Since the $G$ factor is a very sensitive indicator of small scattering contributions from impurities and aggregates, its routine monitoring served as quality control. Anisotropy levels were calculated from the various excitation/emission polarization combinations, taking the $G$ correction factor into account using a macro routine (JASCO) and according to standard equations. ${ }^{5}$ Emission spectra were not corrected for detector and optical path influences. Contributions from Raman scattering were traced and neutralized by parallel measurements with buffer at identical instrument settings and subtracted from all presented emission spectra. Also, Trp-containing solutions (Sigma, Munich, Germany), herewith Trp freely diffusing in solution, were examined. Fluorescence lifetimes, when processing the solutions containing either Trp or hGal-1, were measured by time-correlated single photon counting (TCSPC) using a time-resolved fluorescence spectrometer (FluoTime 200; PicoQuant GmbH, Berlin, Germany) in combination with a pulsed 300 or 340 nm LED (PLS300, PLS340;
PicoQuant GmbH). Viscosity values for sucrose and glycerol solutions were taken from the literature. ${ }^{32,33}$

\section{Results and discussion}

\section{Spectrometric properties of hGal-1}

In the first step, hGal-1 was subjected to measurement of UV absorption spectra as well as fluorescence excitation and emission. Solutions with free Trp were analysed in parallel for internal comparison to the results based on Trp68 present in each of the two subunits of the homodimeric lectin. UV absorption reflected the typical features arising from the two ${ }^{1} \mathrm{~L}_{\mathrm{a}} /{ }^{1} \mathrm{~L}_{\mathrm{b}}$ transition states. ${ }^{5,34}$ The obtained data indicated an increased contribution of the ${ }^{1} \mathrm{~L}_{\mathrm{a}}$ transition for the lectin due to loss of a peak in the rededge part of the spectrum (note involvement of the tyrosine (Tyr)/ phenylalanine (Phe) residues with extinction coefficients of about $1450 \mathrm{M}^{-1} \mathrm{~cm}^{-1}$ at $274 \mathrm{~nm}$ and $200 \mathrm{M}^{-1} \mathrm{~cm}^{-1}$ at $258 \mathrm{~nm}$, respectively, in the blue-edge section) (Fig. 1A). Fig. 1B reports on fluorescence emission at $350 \mathrm{~nm}$ as a function of changes in the excitation wavelength from $200 \mathrm{~nm}$ to $320 \mathrm{~nm}$. In addition to the peak around $280 \mathrm{~nm}$, the position of the absorption maximum, a second peak at $223 \mathrm{~nm}$ was detected. Clear differences between free Trp and galectin were seen in fluorescence emission at the 280 $\mathrm{nm}$ peak. At this wavelength, excitation was performed to record the emission spectra. As shown in Fig. 1C, the position of the maximum for hGal-1 was shifted from that of fully solventexposed Trp (at $355 \mathrm{~nm}$ ) to $344 \mathrm{~nm}$. This result indicated partial contact to an apolar environment because Trp residues located entirely in a protein's interior will yield peaks at less than 330 $\mathrm{nm} .{ }^{5,35}$ Of note, the presence of lactose caused a $5 \mathrm{~nm}$ blue shift, as also reported for ovine Gal-1 using an excitation wavelength of $295 \mathrm{~nm} .{ }^{14}$ Further underscoring the model character of hGal-1, a recent study described similar effects for the $N$-terminal domain of murine Gal-4. ${ }^{36}$ Thus, the interaction with lactose changed the polarity of the environment of the Trp sensor. These results ascertained the reactivity of the lectin and thus set the stage for investigating Trp fluorescence anisotropy.

Steady-state measurements of anisotropy of the Trp fluorescence were performed with a standard VIS polarizer and analyser. They filter the linearly polarized light in the range of 320-750 $\mathrm{nm}$ and result in transmission of less than $5 \%$ in crossed arrangement (see the inset of Fig. 2D). In order to apply standard equipment and minimize contributions from Raman scattering excitation/emission wavelengths of $340 / 425 \mathrm{~nm}$ were set. Moreover, highest anisotropy levels will be reached, when the ${ }^{1} \mathrm{~L}_{\mathrm{a}}$ transition dipole is selectively excited. It is rotated by nearly $90^{\circ}$ relative to the ${ }^{1} \mathrm{~L}_{\mathrm{b}}$ transition dipole so that any contribution from ${ }^{1} \mathrm{~L}_{\mathrm{b}}$ can be precluded at a wavelength larger than $295 \mathrm{~nm} .{ }^{34}$ This setting yielded sufficient Trp-dependent emission at hGal-1 concentrations ranging from 0.1 to $0.5 \mathrm{mg} \mathrm{ml}^{-1}(3-17 \mu \mathrm{M})$ to determine the fluorescence anisotropy $r$ to be around 0.2 (Fig. 2A and $\mathrm{B}$ ). For comparison, the fundamental anisotropy $r_{0}$ is limited to a value of $<0.3 \cdot{ }^{37-39}$ At $300 \mathrm{~nm}$ excitation wavelength, values around 0.26 were reported for Trp-containing peptides and proteins. ${ }^{36,38,39}$ As long as the detected intensity of the fluorescence signal on each polarization channel was sufficiently high (>100 units), data collection was reliable over several days, the standard deviation being about 2\% (ESI, Fig. S1†). To ensure 


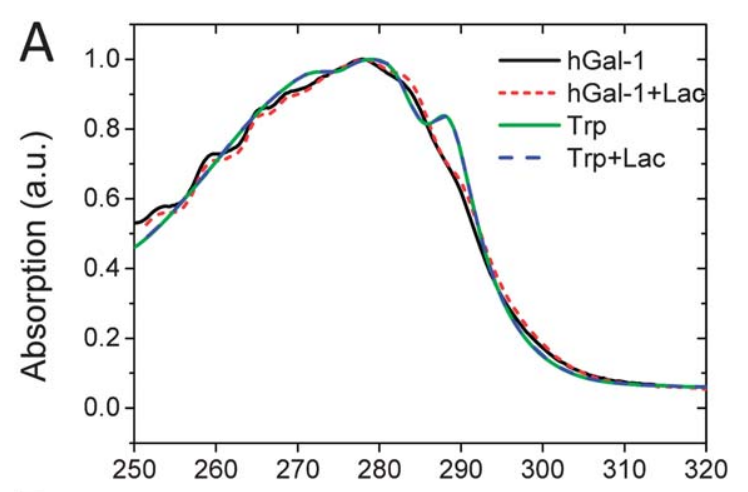

B Wavelength $(\mathrm{nm})$

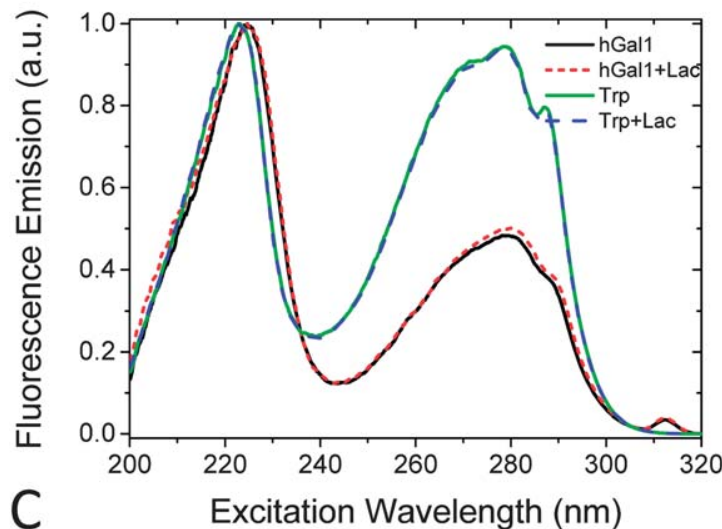

C Excitation Wavelength $(\mathrm{nm})$

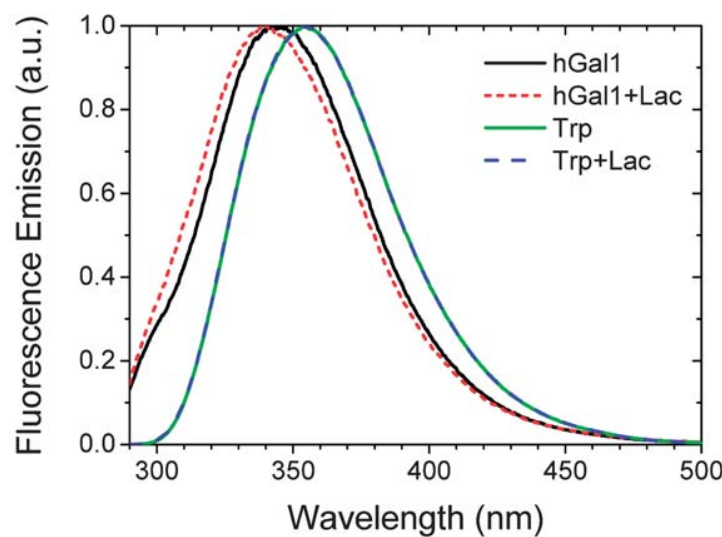

Fig. 1 Trp absorption (A), excitation (B) and emission (C) spectra of free Trp and of hGal-1 in PBS in the absence or presence of $5 \mathrm{mM}$ lactose. Excitation spectra were recorded by monitoring fluorescence emission at $350 \mathrm{~nm}$. Emission spectra were recorded with excitation at $280 \mathrm{~nm}$. All data are normalized to their maximum values.

valid interpretation of the results a major effect by insufficient polarization filters was excluded (ESI, Fig. S2 $\dagger$ ). The same applied to Förster resonance energy transfer because excitation of Tyr/Phe residues was avoided. The intramolecular distance of about $3.9 \mathrm{~nm}$ between the two Trp68 moieties renders a homoTrp transfer (FRET) unlikely.

When adding lactose to the hGal-1-containing solutions, the extent of anisotropy was down-shifted by about 5\% (Fig. 2A and B). Next, the emission wavelength was set to $350 \mathrm{~nm}$ to determine the relationship between anisotropy change and excitation wavelength. A course of signal change typical for indole ${ }^{37-40}$ with a relative minimum between $285 \mathrm{~nm}$ and $290 \mathrm{~nm}$, a local peak around $260 \mathrm{~nm}$ and an increase from $290 \mathrm{~nm}$ onward were recorded. Of note, as also seen for free Trp, Raman scattering at $350 \mathrm{~nm}$ could be detected with an excitation wavelength larger than $305 \mathrm{~nm}$ for hGal-1-containing solutions, causing the anisotropy level to increase. While the polarization states of Trp emission followed a similar dependence on the excitation wavelength for Trp free in solution or presented in hGal-1, the absolute values necessarily differed owing to the disparate levels of rotational freedom. Similarly remarkable, the sign of the anisotropy shift upon ligand binding depended on the selected wavelength for excitation (Fig. 2C and D). Hypothetically, this observation is related to an increased excitation of an oxidized Trp species with fluorescence above $400 \mathrm{~nm} .{ }^{41,42}$ The first galectin detected, i.e. electrolectin from Electrophorus electricus, has been described to be sensitive to oxygen due to Trp-dependent oxidation. ${ }^{11}$ Having detected a consistent effect of lactose on anisotropy, this parameter change was analyzed in quantitative terms.

\section{Ligand binding}

Since an alteration in solvent viscosity would affect translational and rotational diffusion coefficients, it was ascertained first that solvent viscosity was not enhanced by more than $1 \%$ by running experiments for free Trp in PBS containing up to $10 \mathrm{mM}$ lactose (ESI, Fig. S3 $\dagger$ ). This result confirmed a conclusion previously reached based on fluorescence correlation spectroscopy (FCS) data. $^{43}$

By increasing the lactose concentration and measuring the anisotropy level after a sufficient incubation time of more than $0.5 \mathrm{~h}$, the data followed the course of a sigmoidal binding curve (Fig. 3A). It is described by the Hill equation

$$
r\left(c_{\mathrm{L}}\right)=r_{\mathrm{Gal}}+\left(r_{\mathrm{GalL}}-r_{\mathrm{Gal}}\right) \frac{C_{\mathrm{L}}{ }^{n}}{K_{\mathrm{D}}{ }^{n}+C_{\mathrm{L}}{ }^{n}},
$$

where $c_{\mathrm{L}}$ is the ligand concentration, $r_{\mathrm{Gal}}$ is the anisotropy of the ligand-free galectin and $r_{\mathrm{GalL}}$ that of the ligand-loaded galectin, $K_{\mathrm{D}}$ is the equilibrium dissociation constant, and $n$ describes the cooperativity for ligand binding. The observation reported above that the sign of the lactose-induced anisotropy change depends on the excitation/emission wavelengths was confirmed.

Quantitatively, (i) a 5\% increase for the $300 / 350 \mathrm{~nm}$ combination of excitation/emission wavelengths and (ii) a 5\% decrease for the $340 / 425 \mathrm{~nm}$ combination of excitation/emission wavelengths were recorded. The dissociation constants and factors for cooperativity were calculated to be $K_{\mathrm{D}}=(156 \pm 11) \mu \mathrm{M}$ and $n=$ $1.2 \pm 0.1$ in the case (i) and $K_{\mathrm{D}}=(112 \pm 9) \mu \mathrm{M}$ and $n=1.1 \pm 0.1$ in the case (ii) (errors are estimated by the fitting algorithm in the Origin software). These observations are in accord with each other and also with previous estimates for the affinity constant derived from FCS experiments (Table 1). ${ }^{43}$ Using fluorescenceintensity changes upon lactose addition and a one-site model, a $K_{\mathrm{D}}$-value of $(157 \pm 20) \mu \mathrm{M}$ had been reported for the $N$ terminal domain of murine galectin- $4 .^{36}$

Next, the kinetics of binding was followed by successive anisotropy measurements about every 30 seconds for five minute intervals over more than one hour. A representative course of decay of the observed extent of anisotropy that was well fitted by a single-exponential decay model is shown in the inset of Fig. 3B. From such data, relaxation rate constants as functions of the 

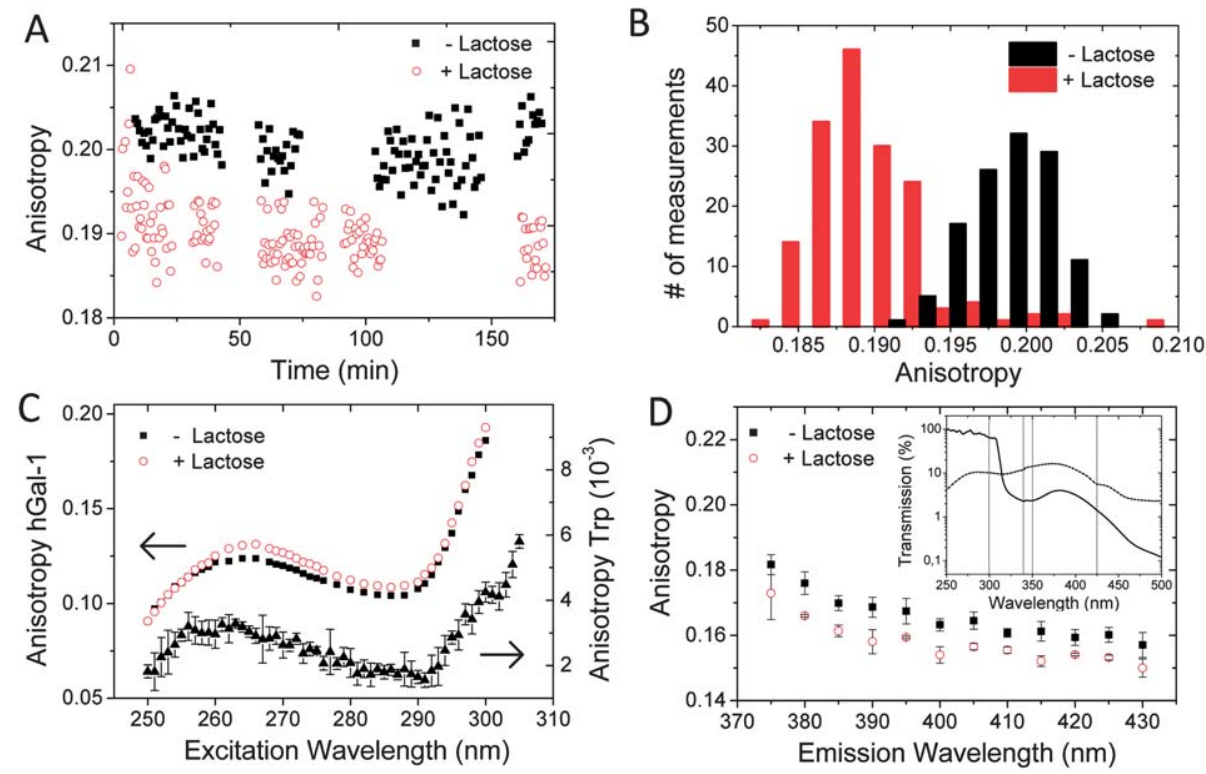

Fig. 2 (A) Trp fluorescence anisotropy of hGal-1 in the presence (open circles) and absence (closed squares) of $5 \mathrm{mM}$ lactose measured with $340 \mathrm{~nm}$ excitation and $425 \mathrm{~nm}$ emission wavelengths over several hours. (B) The same data as in (A) displayed as histogram showing mean $\pm \mathrm{SD}$ of $0.199 \pm 0.003$ and $0.190 \pm 0.004$ for hGal-1 (123 data points, closed bars) and hGal-1 + lactose (162 data points, open bars), respectively. (C) Extent of Trp fluorescence anisotropy of hGal-1 with (open circles) and without (filled squares) $5 \mathrm{mM}$ lactose measured with variable excitation and $350 \mathrm{~nm}$ emission wavelengths. Error bars describing standard deviations are of the same size as the symbols used. For comparison, the anisotropy excitation spectrum of free Trp is shown on a different scale (closed triangles). (D) Extent of Trp fluorescence anisotropy of hGal-1 with (open circles) and without (filled squares) $5 \mathrm{mM}$ lactose measured with $340 \mathrm{~nm}$ excitation and variable emission wavelengths. Error bars represent standard deviations. (Inset) Transmission of two VIS (broken line) or UV (solid line) polarizers in crossed arrangement shown relative to transmission in parallel arrangement. Vertical lines represent the excitation/emission wavelength pairs of $300 / 350 \mathrm{~nm}$ and $340 / 425 \mathrm{~nm}$ used in the various anisotropy experiments.

lactose concentration were obtained, their plotting revealing linear dependence (Fig. 3B). According to theory, the $y$-intercept and the slope represent association and dissociation rate constants, $k_{\mathrm{as}}$ and $k_{\mathrm{dis}}$, for the binding process between hGal-1 and lactose,${ }^{44}$ in numerical values being $k_{\text {as }}=(0.5 \pm 0.1) \mathrm{s}^{-1} \mathrm{M}^{-1}$ together with $k_{\text {dis }}=(1.3 \pm 0.3) \times 10^{-4} \mathrm{~s}^{-1}$. These data are within a factor of five in the range previously determined by FCS..$^{43}$ One reason for the deviation can be that the concentrations tested may not fully satisfy the requirement $c_{\mathrm{Gal}} \ll c_{\mathrm{L}}$ for optimal determination of rate equations, ${ }^{44}$ with a hGal-1 concentration of $1 \mathrm{nM}$ tested in FCS ${ }^{43}$ When calculating the dissociation constant from these rate constants, the resulting $K_{\mathrm{D}}$-value of $(260 \pm 40) \mu \mathrm{M}$ was in reasonable agreement with that derived from kinetic measurements based on FCS and calorimetric titrations, albeit being larger than the $K_{\mathrm{D}}$ estimated by titration assays (FCS/anisotropy) (Table 1). In terms of assay applicability, one-point measurements with test compounds will thus be feasible to reliably trace a reactivity surpassing that of lactose. Having herewith characterized the use of anisotropy measurements for analyzing ligand binding to hGal-1, the question which molecular processes factor into the observed anisotropy values is addressed next. In order to quantify any contribution to depolarization from rotational diffusion or diffusion-independent processes we measured anisotropy as function of solvent viscosity by adding various amounts of glycerol or sucrose to the buffer solution.

\section{Dependence of Trp fluorescence anisotropy on solvent viscosity}

Neither glycerol nor sucrose is expected to interact specifically or strongly with hGal-1. Their presence should therefore modify only diffusion-controlled motion through raising solvent viscosity. Plotting the inverse anisotropy $r^{-1}$ versus inverse viscosity $\eta^{-1}$, a linear dependence described by the Perrin equation ${ }^{5}$

$$
\frac{1}{r}=\frac{1}{r_{0}}+\frac{\tau R T}{r_{0} V \eta}
$$

is thus predicted. In the Perrin equation, which is valid for depolarization by rotational diffusion of spherical particles, the $y$-intercept is described by a viscosity-independent term. Its inverse value is the fundamental anisotropy $r_{0}$ that would be measured at infinitely large solvent viscosity. The slope of eqn (2) is described by the ratio between the fluorescence lifetime $\tau$ times the thermal energy RT, and $r_{0}$ times the molecular volume $V$. For a globular protein, $V$ is proportional to the third power of the hydrodynamic radius $R_{\mathrm{h}}$. The process of depolarization by rotational diffusion of a sphere can also be followed over time.

$$
r(t)=r_{0} \exp \left(-6 D_{\text {rot }} t\right)=r_{0} \exp (-t / \theta)
$$

where the timescale for rotational reorientation is given by the rotational correlation time $\theta$ or the equivalent rotational diffusion constant $D_{\text {rot }}$

$$
D_{\mathrm{rot}}=(6 \theta)^{-1}=\frac{R T}{6 \eta V} .
$$

The collected data indeed reflected the linear behavior described by eqn (2), when the solvent viscosity in hGal-1-containing samples was varied by the addition of glycerol (Fig. 4). A rather weak dependence of the observed extent of anisotropy on solvent viscosity $(\sim 10 \%$ change in anisotropy for a 30 fold 


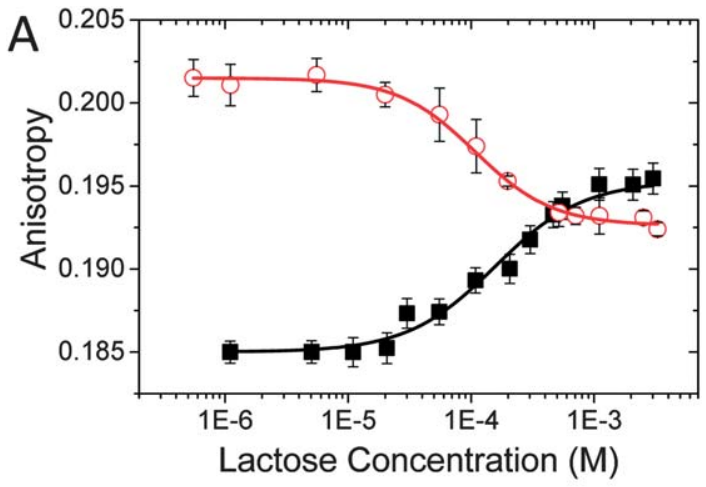

B

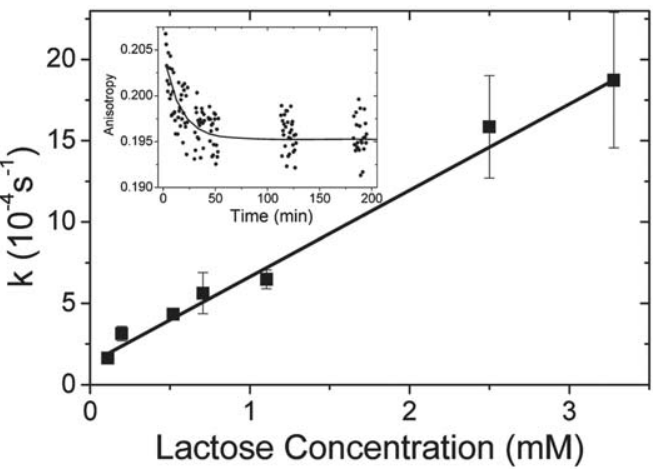

Fig. 3 Binding of lactose to hGal-1 monitored by fluorescence anisotropy. (A) Extent of anisotropy with $300 / 350 \mathrm{~nm}$ excitation/emission (closed squares) or with $340 / 425 \mathrm{~nm}$ (open circles) combinations shown as a function of the lactose concentration and fitted by a Hill model (lines). Error bars represent standard deviations from at least 40 repeated anisotropy measurements. (B) Data of anisotropy measurements over time (observed with the $340 / 425 \mathrm{~nm}$ excitation/emission wavelength combination) are shown in the inset for the lactose concentration of 1 $\mathrm{mM}$. The data curves were fitted by a single-exponential decay. Relaxation rate constants are presented as a function of the concentration of lactose. Error bars represent error estimates from the Origin fitting algorithm.

change in viscosity) was found. The slopes of the linear regression curves in Fig. 4 are $(0.45 \pm 0.03) \mathrm{mPa}$ s for hGal-1 in the absence of lactose and $(0.38 \pm 0.03) \mathrm{mPa}$ s for hGal-1 in the presence of lactose, and the $y$-intercept reached a fairly large value of $4.90 \pm 0.02$, resulting in an apparent fundamental anisotropy of $r_{0}=0.204 \pm 0.001$. The experimentally obtained $r_{0}$ was clearly smaller than $r_{0}$-values of $0.25-0.3$ typically expected for Trp at the given excitation/emission wavelengths. ${ }^{5,36-39}$ This

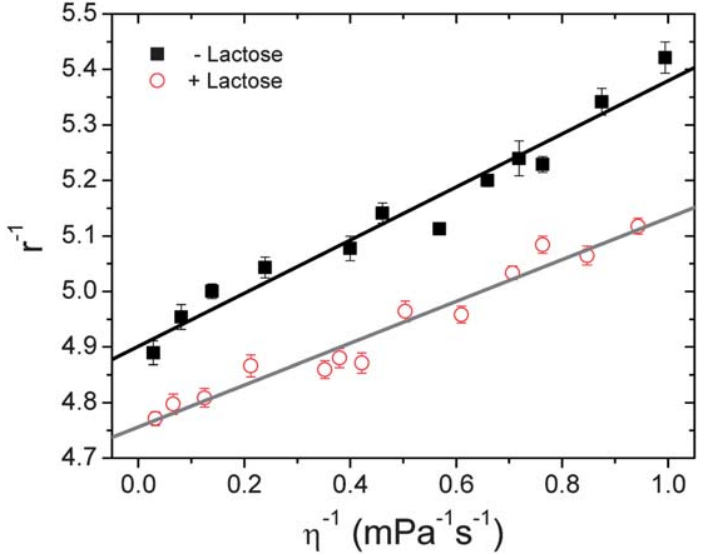

Fig. 4 Viscosity dependence of Trp fluorescence anisotropy for hGal-1 in the absence (filled squares) or presence of $5 \mathrm{mM}$ lactose (open circles) with the $300 / 350 \mathrm{~nm}$ excitation/emission wavelength combination. Inverse anisotropy is shown as a function of inverse viscosity and fitted by linear regression. Viscosity of solutions is varied by the addition of glycerol. Error bars represent standard error of the mean from repeated anisotropy measurements.

finding suggested that the main depolarizing contribution for anisotropy, as measured for hGal-1 in aqueous solution at $20^{\circ} \mathrm{C}$, did not arise merely from rotational diffusion of the protein during the excited-state lifetime. A theoretical estimation of the rotational correlation time $\theta$, which can be calculated for hGal-1 using parameters of its crystal structure (PDBcode: 1GZW) and the computer algorithm HYDROPRO, ${ }^{45}$ turned out to be around $\theta=18 \mathrm{~ns}$ (at $20^{\circ} \mathrm{C}$ in water with a viscosity of $1 \mathrm{cP}$ ). This is more than ten times the fluorescence lifetime of Trp in hGal-1 at $20{ }^{\circ} \mathrm{C}$. In fact, it was determined to be less than $\tau=1.6 \mathrm{~ns}$ (this is the mean relaxation time calculated from the multi-exponential decay, as shown in the ESI, Fig. S4 and Table S1 $\uparrow$ ). According to eqn (2) and (4), the ratio between $\tau$ and $\theta$ would result in an anisotropy ratio

$$
r_{0} / r=1+\tau / \theta
$$

of $r_{0} / r=1.09$ or, in other words, an $8 \%$ decrease in the anisotropy $r$ observed at $20{ }^{\circ} \mathrm{C}$ at $1 \mathrm{cP}$ relative to $r_{0}$. The data, indeed, reached an anisotropy ratio of $r_{0} / r=1.1$.

When repeating experiments in the same set-up in the presence of $5 \mathrm{mM}$ lactose, the same linear behavior according to the Perrin equation with a $-29 \%$ change of the slope $\left(m^{+ \text {lac }}=0.34 \pm 0.02\right.$

Table 1 Binding constants for interaction between hGal-1 and lactose as estimated by various techniques ${ }^{a}$

\begin{tabular}{|c|c|c|c|c|c|}
\hline Method & $K_{\mathrm{D}}$ & $\begin{array}{l}\text { Error } \\
\text { estimate } / 10^{-6} \mathrm{M}\end{array}$ & $n$ & $\begin{array}{l}\text { Error } \\
\text { estimate } / 10^{-6} \mathrm{M}\end{array}$ & Reference \\
\hline Anisotropy $(300 / 350 \mathrm{~nm})$ titration $\left(20^{\circ} \mathrm{C}\right)$ & 156 & 11 & 1.2 & 0.1 & This work \\
\hline FCS titration $\left(20^{\circ} \mathrm{C}\right)$ & 99 & 4 & 1.09 & 0.05 & 43 \\
\hline Anisotropy $(340 / 425 \mathrm{~nm})$ kinetics $\left(20^{\circ} \mathrm{C}\right)$ & 251 & 40 & - & - & This work \\
\hline FCS kinetics $\left(20^{\circ} \mathrm{C}\right)$ & 280 & 90 & - & - & 43 \\
\hline ITC titration $\left(18^{\circ} \mathrm{C}\right)$ & 244 & 6 & - & - & 15 \\
\hline
\end{tabular}

${ }^{a} K_{\mathrm{D}}$ : equilibrium dissociation constant; $n$ : cooperativity factor according to the Hill equation. Titration: $K_{\mathrm{D}}$ and $n$ estimated from a binding curve. Kinetics: $K_{\mathrm{D}}$ estimated from association and dissociation rate constants determined by measurements of binding kinetics. 
versus $\left.m^{-\mathrm{lac}}=0.48 \pm 0.03\right)$ and $\mathrm{a}+3 \%$ change of the apparent fundamental anisotropy $\left(r_{0}{ }^{+ \text {lac }}=0.210 \pm 0.001\right.$ versus $r_{0}{ }^{- \text {lac }}=$ $0.204 \pm 0.001)$ was seen. According to eqn (2) and taking into account that the fluorescence lifetime decreases by $27 \%$ upon binding of lactose (estimated from TCSPC data presented in the ESI, Fig. S3 and Table S1 $\uparrow$ ), the changes in slope translated into a negligible apparent change of the protein's hydrodynamic radius. However, FCS measurements, ${ }^{45}$ together with studies on small angle neutron/X-ray scattering, ${ }^{46}$ had delineated a reduction of the hydrodynamic radius of hGal-1 upon lactose binding by about $-5 \%$. Such an alteration in the hydrodynamic radius will influence both translational and rotational diffusion. ${ }^{47,48}$ Thus, a negative signal change in fluorescence anisotropy is expectable. In contrast, the measured anisotropy increased, and a constant apparent hydrodynamic radius upon lactose binding (with excitation/emission wavelengths of 300/350 nm) appeared to indicate that the alteration in anisotropy could not be caused by an impact on the hydrodynamic radius alone. Since we consistently recorded the depolarization changes, local or socalled segmental motion of the Trp68 residue is likely to accompany and partly compensate the ligand-induced changes in hydrodynamic radius and fluorescence lifetime. Fittingly, such movements had been derived from the NMR-spectroscopical structure analysis of hGal-1 and molecular dynamics simulations, in which Trp68 oriented to the solvent in ligand-free hGal1 was found located within the CRD when in contact to the ligand. ${ }^{49}$ The given approach will thus be a suitable tool to comparatively characterize this structural aspect important for the association process in other galectins.

Turning to sucrose as control for viscosity increase, a deviation from the linear dependence was observed at sucrose quantities above 25\% (w/w) (ESI, Fig. S5A $\dagger$ ). This variation can be attributed to a weak interaction between the protein and sucrose so far not described for hGal-1. Among galectins, evidence for non-lactose binding sites has been given for crystals of the $\mathrm{N}$ terminal domain of murine galectin-4. They show binding sites for glycerol and two units of polyethylene glycol apart from the contact area for lactose but in its vicinity. ${ }^{36}$ The effect of sucrose was even more apparent in a titration series in the presence of 5 $\mathrm{mM}$ lactose (ESI, Fig. S5B $\dagger$ ). The increase in anisotropy upon lactose binding (using excitation/emission wavelengths of 300/ $350 \mathrm{~nm}$ ) could be abolished by sucrose. The use of sucrose gradients for subcellular fractionation may thus impair interaction of hGal-1 to certain counterreceptors.

\section{Conclusions}

The lectin chosen for this study (hGal-1) is representative for a family of clinically relevant proteins, which share key characteristics of the CRD including the central Trp residue. Our measurements establish its polarized fluorescence emission as reliable and sensitive reporter for ligand binding. Fluorescence anisotropy $r$ was about 0.2 , with a change in the presence of lactose by about $5 \%$. Detailed analysis of the depolarization process inferred fast conformational dynamics in the CRD. The underlying segmental motion of the Trp residue will necessarily set limits to the association constant, as reflected by the data. They are indicative of a process much slower than if strictly diffusion controlled. As described, this assay facilitates direct binding experiments in solution without requirement for labeling of lectin or ligand. It therefore is also conveniently suitable for screening compound libraries in the quest to identify potent lectin inhibitors. Moreover, carbohydrate-binding modules and glycoenzymes can similarly be processed, if Trp is involved in ligand contact. Since Tyr can take the place of Trp in carbohydratebinding sites, which explains its respective placement, ${ }^{2,6-8}$ the application of this approach appears reasonable. However, the about 4 fold lower extinction coefficient relative to Trp will have to be compensated for experimentally, along with the consideration of a more frequent presence of Tyr than Trp in proteins.

\section{Abbreviations}

\begin{tabular}{ll}
\hline CRD & carbohydrate recognition domain \\
FCS & fluorescence correlation spectroscopy \\
hGal-1 & human galectin-1 \\
PBS & phosphate-buffered saline \\
TCSPC & time-correlated single photon counting \\
Trp & tryptophan \\
\hline
\end{tabular}

\section{Acknowledgements}

We are grateful to Thorge Holm for accurate characterization and optimization of the polarizing optics, to Dr B. Friday for critical reading of the manuscript, to the reviewers for valuable input as well as for generous funding from the EC Seventh Framework Program (FP7/2007-2013) under grant agreement no. 2602600 ("GlycoHIT").

\section{References}

1 The Sugar Code. Fundamentals of glycosciences, ed. H.-J. Gabius, Wiley-VCH, Weinheim, 2009.

2 H.-J. Gabius, S. André, J. Jiménez-Barbero, A. Romero and D. Solís, Trends Biochem. Sci., 2011, 36, 298-313.

3 M. I. Khan, M. K. Mathew, P. Balaram and A. Surolia, Biochem. J., 1980, 191, 395-400.

4 K. Kakehi, Y. Oda and M. Kinoshita, Anal. Biochem., 2001, 297, 111116.

5 J. R. Lakowicz, Principles of Fluorescence Spectroscopy, Kluwer/ Plenum, New York, 3rd edn, 2006.

6 M. S. Sujatha, Y. U. Sasidhar and P. V. Balaji, Protein Sci., 2004, 13, 2502-2514.

7 J. M. Rini, Annu. Rev. Biophys. Biomol. Struct., 1995, 24, 551-577.

8 C. Taroni, S. Jones and J. M. Thornton, Protein Eng., 2000, 13, 89-98.

9 A. B. Boraston, D. N. Bolam, H. J. Gilbert and G. J. Davies, Biochem. J., 2004, 382, 769-781.

10 P. J. Stein and K. C. Heehn, Biochem. Biophys. Res. Commun., 1980, 95, 547-552.

11 G. Levi and V. I. Teichberg, J. Biol. Chem., 1981, 256, 5735-5740.

12 D. I. Liao, G. Kapadia, H. Ahmed, G. R. Vasta and O. Herzberg, Proc. Natl. Acad. Sci. U. S. A., 1994, 91, 1428-1432.

13 H.-C. Siebert, R. Adar, R. Arango, M. Burchert, H. Kaltner, G. Kayser, E. Tajkhorshid, C.-W. von der Lieth, R. Kaptein, N. Sharon, J. F. G. Vliegenthart and H.-J. Gabius, Eur. J. Biochem., 1997, 249, 27-38.

14 M. M. Iglesias, G. A. Rabinovich, V. Ivanovic, C. Sotomayor and C. Wolfenstein-Todel, Eur. J. Biochem., 1998, 252, 400-407.

15 M. F. López-Lucendo, D. Solís, S. André, J. Hirabayashi, K.-i. Kasai, H. Kaltner, H.-J. Gabius and A. Romero, J. Mol. Biol., 2004, 343, 957-970.

16 A. Villalobo, A. Nogales-González and H.-J. Gabius, Trends Glycosci. Glycotechnol., 2006, 18, 1-37. 
17 S. Rorive, N. Belot, C. Decaestecker, F. Lefranc, L. Gordower, S. Micik, C.-A. Maurage, H. Kaltner, M.-M. Ruchoux, A. Danguy, H.-J. Gabius, I. Salmon, R. Kiss and I. Camby, Glia, 2001, 33, 241255.

18 O. Roda, E. Ortiz-Zapater, N. Martínez-Bosch, R. GutiérrezGallego, M. Vila-Perelló, C. Ampurdanés, H.-J. Gabius, S. André, D. Andreu, F. X. Real and P. Navarro, Gastroenterology, 2009, 136, 1379-1390.

19 J. Wang, Z.-H. Lu, H.-J. Gabius, C. Rohowsky-Kochan, R. W. Ledeen and G. Wu, J. Immunol., 2009, 182, 4036-4045.

20 S. André, C. E. P. Maljaars, K. M. Halkes, H.-J. Gabius and J. P. Kamerling, Bioorg. Med. Chem. Lett., 2007, 17, 793-798.

21 S. André, D. Giguère, T. K. Dam, C. F. Brewer, H.-J. Gabius and R. Roy, New J. Chem., 2010, 34, 2229-2240.

22 T. Purkrábková, K. Smetana Jr, B. Dvořánková, Z. Holíková, C. Böck, M. Lensch, S. André, R. Pytlík, F.-T. Liu, J. Klíma, K. Smetana, J. Motlík and H.-J. Gabius, Biol. Cell, 2003, 95, 535-545.

23 S. André, H. Sanchez-Ruderisch, H. Nakagawa, M. Buchholz, J. Kopitz, P. Forberich, W. Kemmner, C. Böck, K. Deguchi, K. M. Detjen, B. Wiedenmann, M. von Knebel Doeberitz, T. M. Gress, S.-I. Nishimura, S. Rosewicz and H.-J. Gabius, FEBS J., 2007, 274, 3233-3256.

24 D. Solís, M. J. Maté, M. Lohr, J. P. Ribeiro, L. López-Merino, S. André, E. Buzamet, F. J. Cañada, H. Kaltner, M. Lensch, F. M. Ruiz, G. Haroske, U. Wollina, M. Kloor, J. Kopitz, J. L. Sáiz, M. Menéndez, J. Jiménez-Barbero, A. Romero and H.-J. Gabius, Int. J. Biochem. Cell Biol., 2010, 42, 1019-1029.

25 H.-J. Gabius, R. Engelhardt, F. Cramer, R. Bätge and G. A. Nagel, Cancer Res., 1985, 45, 253-257.

26 S. André, Z. Pei, H.-C. Siebert, O. Ramström and H.-J. Gabius, Bioorg. Med. Chem., 2006, 14, 6314-6326.

27 S. André, D. Specker, N. V. Bovin, M. Lensch, H. Kaltner, H.-J. Gabius and V. Wittmann, Bioconjugate Chem., 2009, 20, 1716-1728.

28 J. Kopitz, M. Bergmann and H.-J. Gabius, IUBMB Life, 2010, 62, 624-628.
29 J. T. Powell and P. L. Whitney, Biochem. J., 1984, 223, 769-774.

30 P. L. Whitney, J. T. Powell and G. L. Sanford, Biochem. J., 1986, 238, 683-689.

31 T. K. Dam, H.-J. Gabius, S. André, H. Kaltner, M. Lensch and C. F. Brewer, Biochemistry, 2005, 44, 12564-12571.

32 M. L. Sheely, Ind. Eng. Chem., 1932, 24, 1060-1064.

33 M. Mathlouthi and J. Génotelle, in Sucrose, Properties and Applications, eds M. Mathlouthi and P. Reiser, Blackie Academic \& Professional, London, 1995, pp. 126-154.

34 P. R. Callis, Methods Enzymol., 1997, 278, 113-150.

35 J. T. Vivian and P. R. Callis, Biophys. J., 2001, 80, 2093-2109.

36 V. Krejčiríiková, P. Pachl, M. Fábry, P. Malý, P. Řezáčová and J. Brynda, Acta Crystallogr., Sect. D: Biol. Crystallogr., 2011, 67, 204-211.

37 B. Valeur and G. Weber, Photochem. Photobiol., 1977, 25, 441-444.

38 J. R. Lakowicz, B. P. Maliwal, H. Cherek and A. Balter, Biochemistry, 1983, 22, 1741-1752.

39 G. Weber, Biochem. J., 1960, 75, 335-345.

40 G. Weber, Biochem. J., 1960, 75, 345-352.

41 A. Pirie, Biochem. J., 1972, 128, 1365-1367.

42 G. E. Ronsein, M. C. B. Oliveira, S. Miyamoto, M. H. G. Medeiros and P. D. Mascio, Chem. Res. Toxicol., 2008, 21, 1271-1283.

43 A. Göhler, S. André, H. Kaltner, M. Sauer, H.-J. Gabius and S. Doose, Biophys. J., 2010, 98, 3044-3053.

44 H. Gutfreund, Kinetics for the Life Sciences: Receptors, Transmitters and Catalysts, Cambridge University Press, Cambridge, UK, 1995.

45 J. Garcia De La Torre, M. L. Huertas and B. Carrasco, Biophys. J., 2000, 78, 719-730.

46 L. He, S. André, H.-C. Siebert, H. Helmholz, B. Niemeyer and H.-J. Gabius, Biophys. J., 2003, 85, 511-524.

47 J. M. Tsay, S. Doose and S. Weiss, J. Am. Chem. Soc., 2006, 128, 1639-1647.

48 A. Einstein, Ann. Phys., 1905, 17, 549-560.

49 I. V. Nesmelova, E. Ermakova, V. A. Daragan, M. Pang, M. Menéndez, L. Lagartera, D. Solís, L. G. Baum and K. H. Mayo, J. Mol. Biol., 2010, 397, 1209-1230. 